\title{
Design and Determination of Optimum Coefficients of IIR Digital Highpass Filter using Analog to Digital Mapping Technique
}

\author{
Subhadeep Chakraborty \\ Department of ECE \\ Calcutta Institute of Technology \\ Uluberia, Howrah, West Bengal, \\ India, PIN-711316
}

\author{
Subhasish Maitra \\ Department of ECE \\ Kalyani Govt. Engg. College \\ Kalyani, Nadia.
}

\begin{abstract}
In Digital Signal Processing (DSP), there two types of filters are used to perform the filtering operations and they are the Infinite Impulse Response (IIR) filter and the Finite Impulse Response (FIR) filter. The present output sample of an IIR filter depends on the present input samples, past input samples and past output samples, i.e IIR filter is of recursive type. Now to design the Digital IIR filter, the coefficients are essentially required. There are a numbers of techniques available for designing the IIR filter. In this paper, the design and determination of the IIR filter coefficients are introduced using computer based approach. The program based on the algorithm proposed in this paper is simulated in Matlab which provides with the satisfactory results.
\end{abstract}

Keywords: IIR filter, Digital filters, coefficient, High pass filter, Butterworth filter, Chebyshev filter, analog-to-digital mapping.

\section{Introduction}

In Digital Signal Processing (DSP) the filters are very essential because they are practically introduced to filter out the desired frequency as per requirement to be used in different area of interest, for example Communication Engineering i.e. to process the desired signal fromone point to another. In that case, the total band of frequency is transmitted through the transmitter and the user select the desired frequency to get the particular information, for example Radio receivers. Now to select the proper frequency band, the filter is used[1][2][3].

In DSP, there are typically two systems. The first one, i.e. the Digital filters, which performs the filtering operation in time domain[4][5][7]. The second one is the Spectrum analyzer, which represents the sihnal in frequency domain[4][5][21]. The impulse response of an IIR filter is of infinite duration whereas the impulse response of a FIR filter is of finite duration. The response of an IIR filter is much better than that of a FIR filter having same numbers of coefficients[8][16].

There are certain properties of IIR filter such as pass-band width, stop-band width, maximum allowable pass-band and stop-band ripples.[4][5][8][16]. Generally the filters are designed with resistor, capacitor and op-amps to get the desired filtering operation because the op-amp is able to show the recursive effect and so it is very useful in designing the IIR filter as it is actually a recursive filter. whereas the FIR filter is non recursive filter[5][8][16]. The IIR filter that is designed with the resisters, capacitors and op-amp is actually an Analog filter. That is the transfer function we will get from this type of filter is the transfer function in analog plane. The digital filter have several features such as high accuracy and reliability, small physical size and reduced sensitivity to component tolerances or drift[4][9][13]. So to get the transfer function in digital plane or in other words to design a digital filter from the predesigned analog filter, we need to introduce the frequency transformation. So for this purpose, the analog to digital frequency transformation technique is applied, is called the analog to digital mapping technique.[4][5][6][7].

\section{Design of IIR Filter}

The IIR filter can be designed by active or passive elements as described in the introductory part. Now there are many type of IIR filters such as Butterworth filter, Chebyshev filter, Elliptic filter etc[4][8][14][15]. The design of the IIR filters are shown from Fig.1 to fig.2[4][9][10].

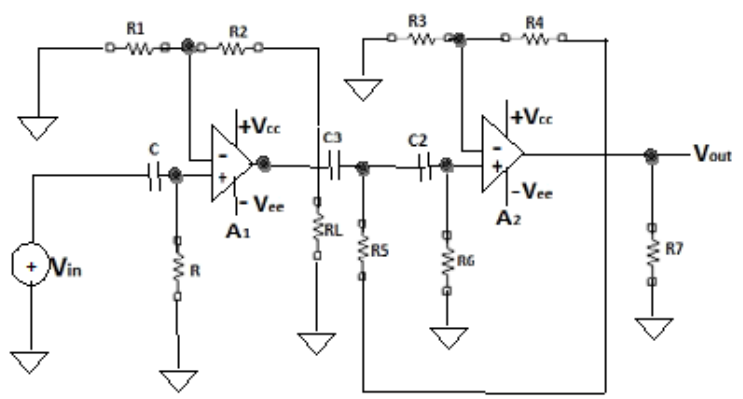

Fig.1 IIR High pass Butterworth Filter

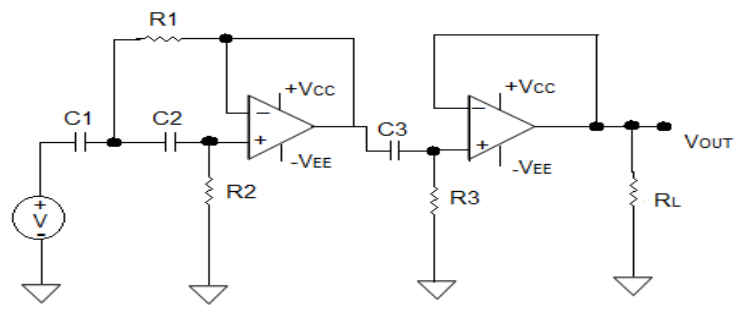

Fig.2 IIR High pass Chebyshev Filter 
In DSP, in case of filter design, when the components like resistors, capacitors etc are connected, this is referred to as a passive filter until and unless the active components like voltage source is connected. When a voltage source is connected with that passive filter circuit, the filter becomes a active filter[9][11]. At the time of observation of the output response of filter, the order of FIR filter is higher than that of the IIR filter for the same magnitude response. So to reduce the order of a filter and to reduce the signal processing time, it is important to design the IIR filter rather than designing FIR filter[8].

Fig.1 and Fig.2 shows the design of Butterworth filter and Chebyshev filter respectively. Basically the available filter orders are $1^{\text {st }}$ order filter and $2^{\text {nd }}$ order filter. Now by cascading these two, the several high order filters can be designed. For example, if the $9^{\text {th }}$ order filter will have to be designed, the fashion will be the cascading of four $2^{\text {nd }}$ order filter and one $1^{\text {st }}$ order filter[9]. The higher the order of a filter, the better in the response.

After designing the analog filter, the pole-zero locations as well as the stability of the filter must be determined. In that case, the analog filter must be converted to an equivalent digital filter with the application of analogto-digital mapping technique. There are many methods for this mapping, for example Bilinear transformation, Impulse invariance method[8][16][21]. After successful mapping, the transfer function of the digital filter can be obtained or in other words the filter coefficients can be calculated which is one of the most important for digital filter design[4][5][18][20]. In case of analog filter, the transfer function can be found in s-plane but for digital filter, the transfer function is obtained in z-plane[10][11].

\section{Realization of IIR Digital Filter}

IIR Filter has a network or system which is of recursive type. Such recursive system can be described by the following Difference equation where $\mathrm{x}(\mathrm{n})$ is the input of the system and $y(n)$ is the output of that system[8][16][17],

$$
\begin{aligned}
\begin{aligned}
y(n)= & -\sum_{k=1}^{N} a_{k} y(n-k)+\sum_{k=0}^{M} b_{k} x(n-k) \ldots . .(1.1) \\
y(n)= & -a_{1} y(n-1)-a_{2} y(n-2) \ldots \ldots-a_{N-1} y(n-N+1) \\
& -a_{N} y(n-N)+b_{0} x(n)+b_{1} x(n-1) \\
& \ldots . . .+b_{M} x(n-M) \\
& \text { Let, } \\
w(n)= & b_{0} x(n)+b_{1} x(n-1) \ldots . . .+b_{M} x(n-M)
\end{aligned}
\end{aligned}
$$

Equation (1.1a) can be represented as,

$$
\begin{array}{r}
y(n)=-a_{1} y(n-1)-a_{2} y(n-2) \ldots \ldots-a_{N-1} y(n-N+1) \\
-a_{N} y(n-N)+w(n) \quad \ldots \ldots(1.1 \mathrm{c})
\end{array}
$$

Now taking the Z-Transform in the both sides of equation (1.1a), we get

$$
\begin{aligned}
Y(z)= & -a_{1} z^{-1} Y(z)-a_{2} z^{-2} Y(z) \ldots \ldots-a_{N-1} z^{-(N-1)} Y(z) \\
& -a_{N} z^{-N} Y(z)+b_{0} X(z)+b_{1} z^{-1} X(z) \ldots \ldots \\
& \ldots .+b_{M} z^{-M} X(z)
\end{aligned}
$$

Rearranging the equation (1.2), we get,

$$
\begin{aligned}
Y(z)= & -\left(a_{1} z^{-1}+a_{2} z^{-2} \ldots \ldots . .+a_{N-1} z^{-(N-1)}+a_{N} z^{-N}\right) Y(z) \\
& +\left(b_{0}+b_{1} z^{-1} \ldots \ldots \ldots+b_{M} z^{-M}\right) X(z) \quad \ldots(1.2 \mathrm{a})
\end{aligned}
$$

So, from the Equation (1.1a) it is clear that in a IIR system, the present output depends upon the past inputs and the past outputs. Now taking the Z-Transform of Equation (1.1a) the structure of the IIR Filter can be determined with the application of Direct form-I Realization. This method is used to separate delays for both input and output. This realization requires $\mathrm{M}+\mathrm{N}+1$ multiplications, $\mathrm{M}+\mathrm{N}$ additions and $\mathrm{M}+\mathrm{N}+1$ memory locations. There are various form of realizations[8] of an IIR Filter such as,

1. Direct form - I realization

2. Direct form - I realization

3. Transposed direct form realization

4. Cascade form realization

5. Parallel form realization

6. Lattice-Ladder structure realization

The structure of Direct form - I realization for an IIR Filter described in equation (1.1a) is shown in Fig.3 [ 8][16][19].

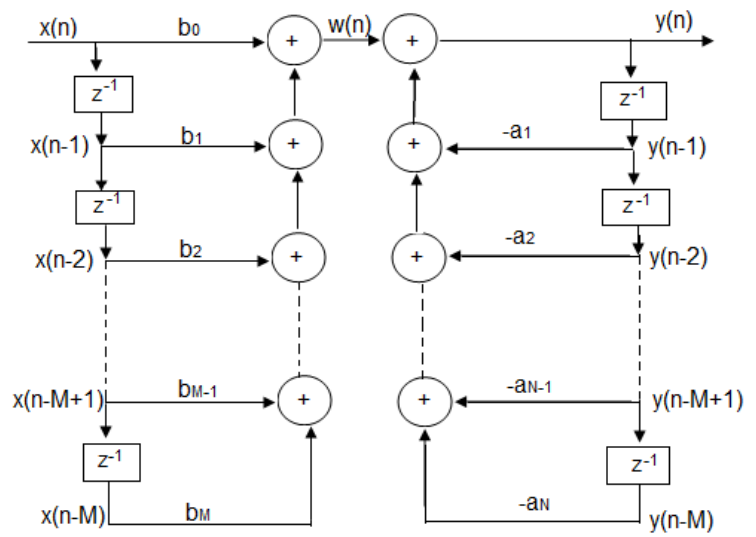

Fig.3 Direct Form-I realization of IIR Digital filter

\section{Determination of the Transfer Function}

The impulse response is actually the ratio of the produced output to the given input, that is more elaborately, the transfer function $\mathrm{H}(\mathrm{z})$ is actually the ratio of the ZTransform of output to the input.

The impulse response $h(n)$ for a realizable filter is [8][16],

$$
h(n)=0 \quad \text { for } n \leq 0
$$

where $h(n)$ must satisfy the following condition to ensure the stability of a filter[8][16],

$$
\sum_{n=0}^{\infty}|h(n)|<\infty
$$

Now, the Z-Transform of the impulse response $h(n)$ i.e. $\mathrm{H}(\mathrm{z})$ is referred to as the transfer function of a digital filter. So, our interest goes to the determination of the $\mathrm{H}(\mathrm{z})$ because the $\mathrm{H}(\mathrm{z})$ contains the numerator and denominator 
coefficients which are essentially required to design the Digital filter.

Now to determine the transfer function of the IIR filter, we rewrite the equation (1.2a) and get the required $\mathrm{H}(\mathrm{z})$ equation i.e.,

$$
\begin{aligned}
& \boldsymbol{H}(z)=\frac{\sum_{n=0}^{M} \boldsymbol{b}(\boldsymbol{n}) z^{-n}}{\mathbf{1}+\sum_{n=1}^{N} \boldsymbol{a}(\boldsymbol{n}) z^{-n}} \\
& =\frac{B(z)}{A(z)}=\frac{b(0)+b(1) z^{-1}+b(2) z^{-2}+\ldots \ldots .+b(M) z^{-M}}{1+a(1) z^{-1}+a(2) z^{-2}+\ldots \ldots \ldots \ldots .+a(N) z^{-N}}
\end{aligned}
$$

Where,

$b(n)=$ Numerator coefficient of the filter $\mathrm{a}(\mathrm{n})=$ Denominator coefficient of the filter $\mathrm{H}(\mathrm{z})=\mathrm{Y}(\mathrm{z}) / \mathrm{X}(\mathrm{z})=\mathrm{B}(\mathrm{z}) / \mathrm{A}(\mathrm{z})=$ Transfer function

After successful determination of the transfer function, we can calculate the coefficients of the filter i.e. the determination of $b(n)$ and $a(n)$. Actually the Transfer Function refers that for giving some input how much output can be obtained. There are various techniques available to design and calculate the coefficients of an IIR Digital high pass filter. The algorithm referred here can be used for smarter determination of IIR Digital high pass filter and is much more helpful for the determination of the transfer function as well as the coefficients. The flowchart is given in Fig.4.

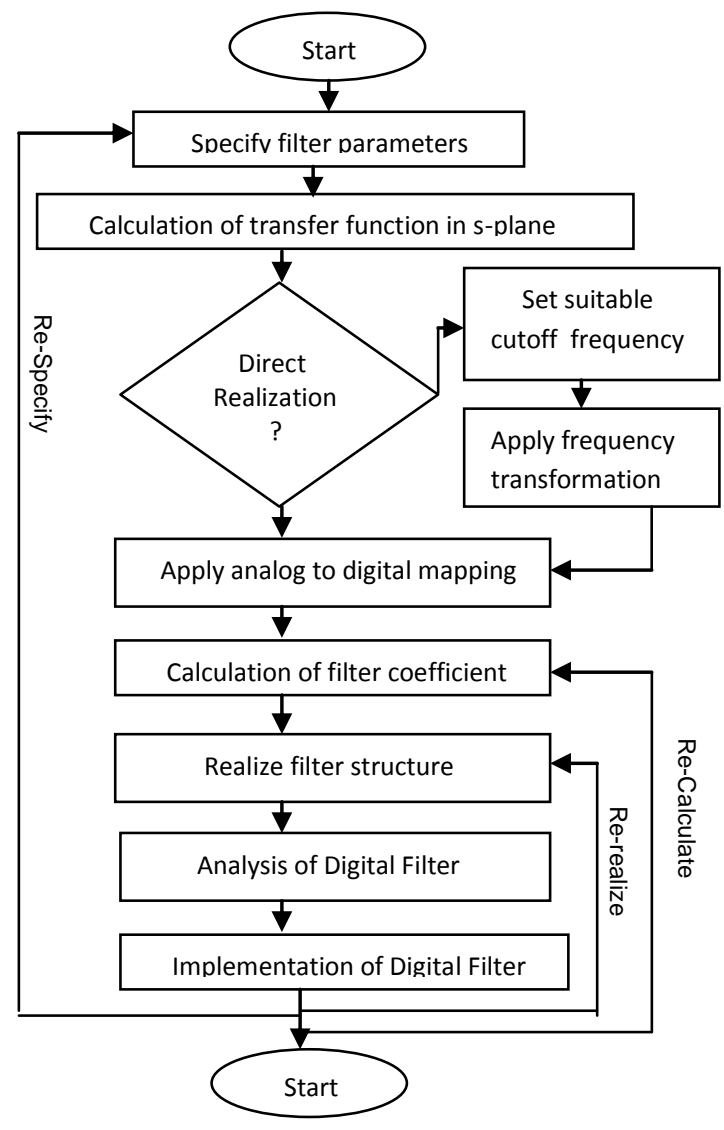

Fig.4 Proposed algorithm
Now, applying the proposed algorithm and specifying the necessary parameters, the designer can suitably design the IIR Digital high pass filter. The algorithm has a special feature that it provides the user with two techniques of realization of IIR Digital high pass filter

\section{Direct realization \\ 2. Indirect realization}

In indirect realization[4], based on the specified cutoff frequency, the IIR Digital high pass filter can be designed by using the frequency transformation technique.

In the direct method of realization[4], first the transfer function of an analog filter is to be calculated, i.e. the transfer function in s-plane. Then by applying the analog-to-digital mapping technique, the transfer function in z-plane is obtained. Now, the transfer function that is obtained in z-plane is actually the transfer function of the digital filter.

So, first we design the network for the IIR Digital high pass filter, then realize the structure of that and after that by applying the analog-to-digital mapping technique i.e. s-plane to z-plane transformation, we finally achieved the transfer function in z-plane or in other words, the transfer function of the predesigned IIR Digital high pass filter.

\section{S-plane to Z-plane transformation}

S-plane to z-plane transformation simply means that analog-to-digital mapping where the stable poles of s-plane can be successfully mapped into z-plane. Fig. 1 shows a IIR filter network. If we perform the Laplace transform on the impulse response $\mathrm{h}(\mathrm{t})$ of that network, we get the transfer function $\mathrm{H}(\mathrm{s})$ in s-plane i.e.[8][10] ,

$$
H(s)=L\{h(t)\}=\int_{0}^{\infty} h(t) \cdot e^{-s t} d t
$$

where,

$$
\begin{aligned}
\mathrm{s} & =\text { complex variable } \\
& =\sigma+\mathrm{j} \Omega
\end{aligned}
$$

Now, to get the discrete signal $h(n)$ from the $h(t)$, we substitute ,

$$
\mathrm{t}=\mathrm{nT}
$$

where,

$\mathrm{T}=$ sampling time

If $\mathrm{T}=1 \mathrm{sec}$, then we get $\mathrm{t}=\mathrm{n}$ from equation (1.7). After substitution, we can easily achieve $h(n)$ from $h(t)$ where $h(n)$ is the discrete form of the time domain continuous signal $h(t)$. If we perform the $\mathrm{z}$-transform on $\mathrm{h}(\mathrm{n})$, we can get the transfer function in z-plane i.e. H(z)[8][16],

$$
H(z)=Z\{h(n)\}=\sum_{n=-\infty}^{\infty} h(n) z^{-n}
$$

So, in this way, the transfer function of an IIR Digital filter can be obtained. Basically the relation between the splane and z-plane [8] can be described by the equation,

$$
z=e^{s T}
$$


Let,

$$
z=r e^{j \omega}
$$

and,

$$
s=\sigma+j \Omega
$$

Now, substituting equation (1.10) and (1.6) into equation (1.9), we get,

$$
z=e^{s T}=e^{(\sigma+j \Omega) T}=e^{\sigma T} e^{j \Omega T}
$$

Comparing equation (1.10) and (1.11), we get that,

$$
|z|=e^{\sigma T}
$$

and

$$
\omega=\Omega T
$$

We can consider three cases to determine the values of $|z|$ with respect to the values of $\sigma$ as follows[8],

Case I: $\quad \sigma=0$ gives $|z|=1 \rightarrow$ Stable system Case II: $\sigma<0$ gives $|\mathrm{z}|<1 \rightarrow$ Stable system Case III: $\sigma>0$ gives $|z|>1 \rightarrow$ Unstable system

For the value of $\sigma=0$ and $\sigma<0$, the $j \Omega$ axis of s-plane maps into the unit circle on the $z$-plane and for $\sigma>0$, The right half of s-plane maps outside the unit circle on the z-plane. So, to ensure the stability of the system, we must consider the values of $\sigma$ as $\sigma<0$ and $\sigma=0$. The Fig. 5 shows the concept of s-plane to z-plane mapping for stable system and the Fig.6 shows the mapping for unstable system[8].

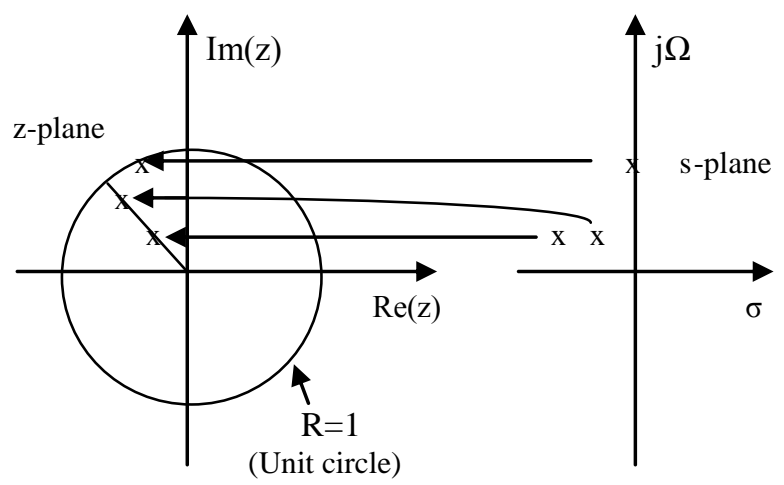

Fig.5 Mapping of poles from s-plane to z-plane ( For stable system)

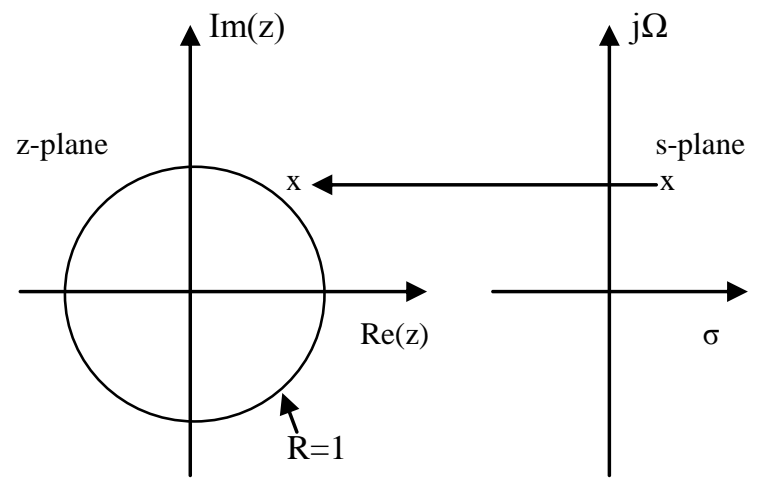

Fig.6 Mapping of poles from s-plane to z-plane ( For unstable system)

\section{Comparison of Butterworth and Chebyshev Filter}

Butterworth filter and Chebyshev filter both are used for the high frequency filtering or low frequency filtering operation. These two filters are used in some specific area of interests. So, there are the reason where we use the Butterworth filter and where the Chebyshev filter. In some cases, the Butterworth filter is better than the Chebyshev filter and vice versa. Which filter will be used in which area, depends upon their properties such as magnitude response, pole locations, order of filter etc. Here is some comparisons in between these two filters[8][12],

- The magnitude response of the Butterworth filter decreases monotonically as the frequency increases from 0 to $\infty$ whereas the magnitude response of the Chebyshev filter exhibits ripples in the passband or stopband depending upon the type. Chebyshev Type-I filter exhibits equiripple behavior in passband and Chebyshev Type-II filter exhibits that of in stopband.

- The poles of Butterworth filter lies on a circle whereas the poles of the Chebyshev filter lies on an ellipse.

- Butterworth filter contains poles and zeros both but Chebyshev Type-I filter contains only poles and for that reason Chebyshev Type-I filter is called All Pole Filter.

- The transition band is more in Butterworth filter compared to the Chebyshev filter.

- For the same filter parameter specification, the number of poles are more in Butterworth filter than Chebyshev filter. This indicates that, with same specifications, the order of Butterworth filter is higher than that of the Chebyshev filter. That means, to construct the Chebyshev filter, less numbers of discrete components are required compared to the Butterworth filter. 


\section{Simulation result}

The programs to design the IIR Butterworth Highpass filter and IIR Chebyshev Highpass filter(Type-I and Type-II) are simulated in Matlab7. The proper specifications, such as the passband frequency, the stop band frequency, the passband and stopband ripples, are chosen in those programs, to have the perfect result in the magnitude response, phase response, impulse response and pole-zero plotting and moreover for the coefficient determination. The pole-zero plotting shows the stability of the system. The output graphs are shown from Fig.7 to Fig.22.

Table 1 gives us the result of the coefficients for IIR Butterworth Highpass filter and IIR Chebyshev Highpass filter(Type-I and Type-II) for order $=4$ and order $=6$.

\begin{tabular}{|c|c|c|c|c|}
\hline $\begin{array}{l}\text { Filter } \\
\text { name }\end{array}$ & $\begin{array}{l}\text { Filter } \\
\text { order }\end{array}$ & Type & $\begin{array}{l}\text { Numerator } \\
\text { coefficient }\end{array}$ & $\begin{array}{l}\text { Denominator } \\
\text { coefficient }\end{array}$ \\
\hline \multirow{2}{*}{$\begin{array}{l}\text { Butterworth } \\
\text { Highpass } \\
\text { Filter }\end{array}$} & 4 & \multirow[b]{2}{*}{ N/A } & $\begin{array}{l}0.6531,0.461 \\
0.122,0.01436\end{array}$ & $\begin{array}{l}0.3731,-0.8696 \\
1.304,-1.348\end{array}$ \\
\hline & 6 & & $\begin{array}{l}1.806,1.764, \\
0.7177,0.1558, \\
0.01902, \\
0.001239\end{array}$ & $\begin{array}{l}0.9612,-2.601 \\
4.399,-5.155 \\
4.454,-2.729\end{array}$ \\
\hline \multirow{4}{*}{$\begin{array}{l}\text { Chebyshev } \\
\text { Highpass } \\
\text { Filter }\end{array}$} & 4 & \multirow[t]{2}{*}{ I } & $\begin{array}{l}0.01281, \\
-0.0297,0.0259, \\
-0.01003\end{array}$ & $\begin{array}{l}0.2972,-1.545 \\
3.091,-2.827\end{array}$ \\
\hline & 6 & & $\begin{array}{l}0.00071, \\
-0.0025,0.0037, \\
-0.0029,0.0013, \\
-0.0031\end{array}$ & $\begin{array}{l}0.1342,-1.081 \\
3.681,-6.795 \\
7.173,-4.11\end{array}$ \\
\hline & 4 & \multirow{2}{*}{ II } & $\begin{array}{l}1.245,-4.358 \\
6.085,-2.922\end{array}$ & $\begin{array}{l}1.253,-4.358 \\
6.085,-3.931\end{array}$ \\
\hline & 6 & & $\begin{array}{l}1.021,-5.277 \\
11.88,-14.74 \\
10.57,-4.137\end{array}$ & $\begin{array}{l}1.278,-6.825 \\
15.79,-20.06 \\
14.7,-5.875\end{array}$ \\
\hline
\end{tabular}

\section{i) Magnitude response (Filter order $=4$ )}

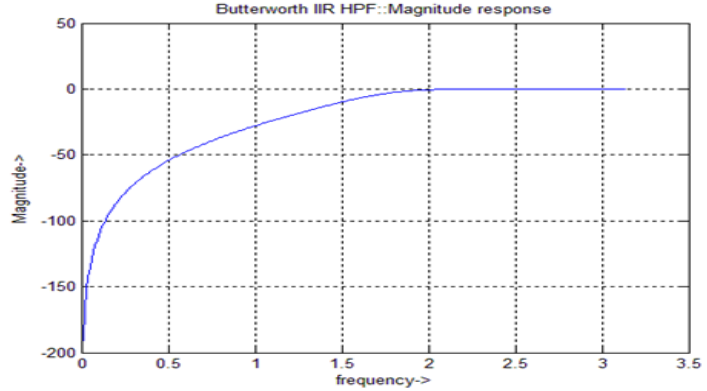

Fig.7 Butterworth Highpass filter Magnitude response

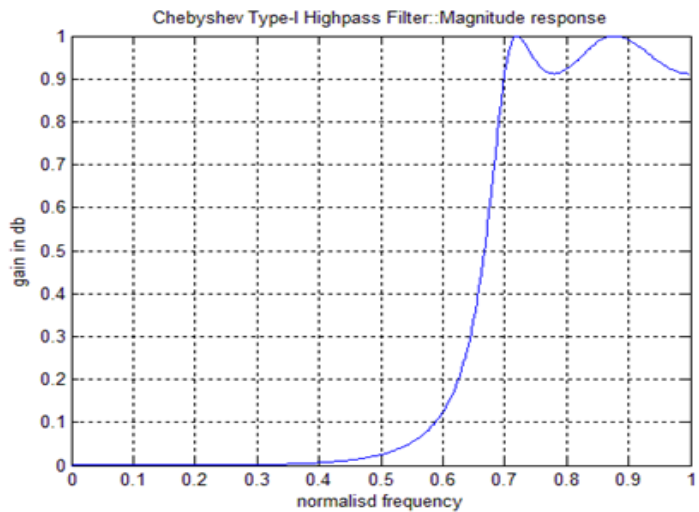

Fig.8Chebyshev(Type-I) Highpass filter Magnitude response

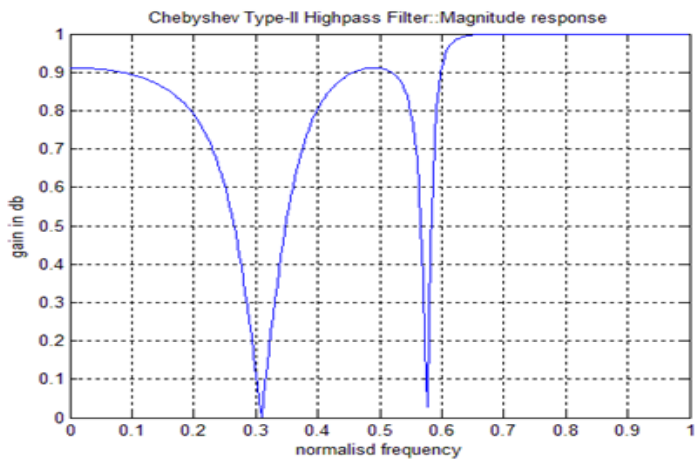

Fig.9Chebyshev(Type-II) Highpass filter Magnitude response

\section{ii) Impulse response(Filter order $=4$ )}

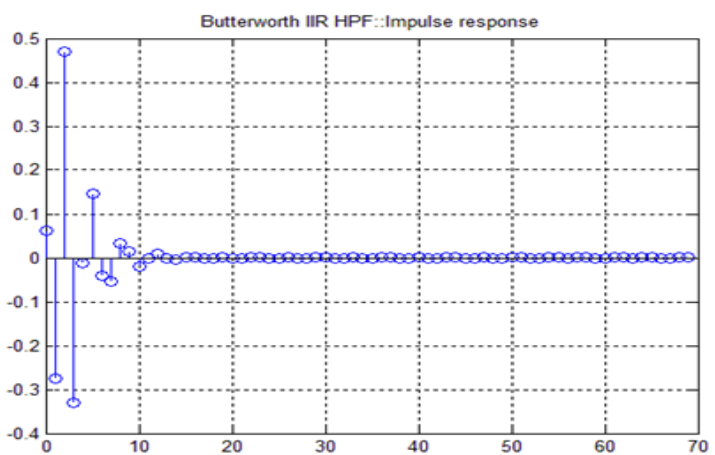

Fig.10 Butterworth Highpass filter Impulse response

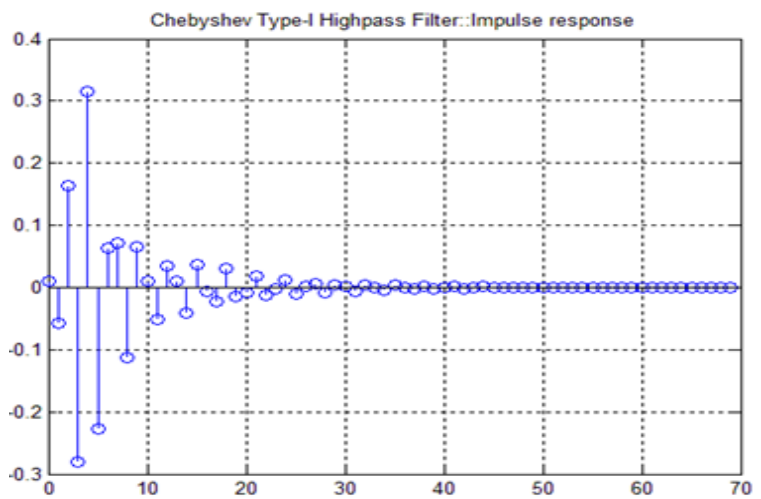

Fig.11Chebyshev(Type-I) Highpass filter Impulse response 


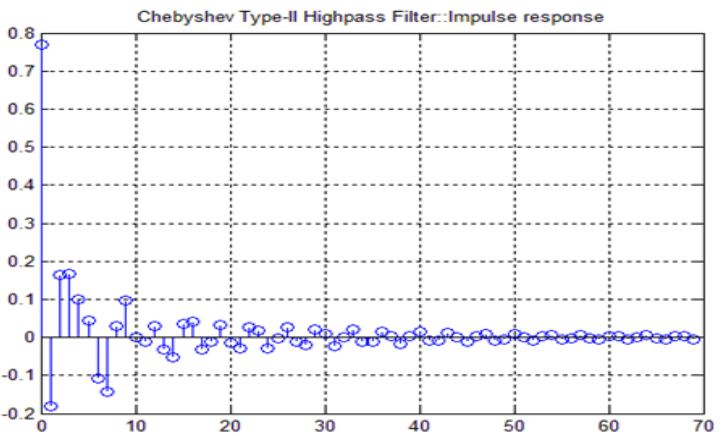

Fig.11Chebyshev(Type-II) Highpass filter Impulse response

\section{iii) Pole-Zero plot (Filter order $=4$ )}

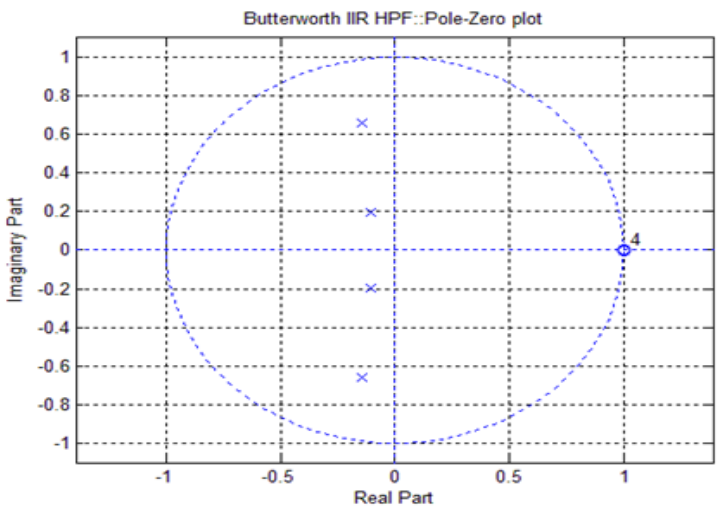

Fig.12 Butterworth Highpass filter pole-zero plot

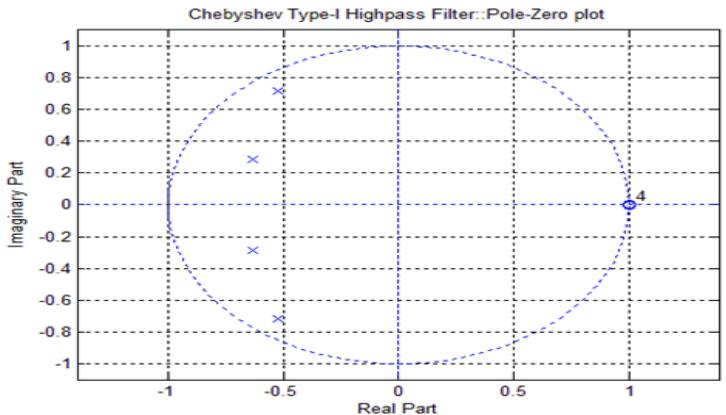

Fig.13Chebyshev(Type-I) Highpass filter pole-zero plot

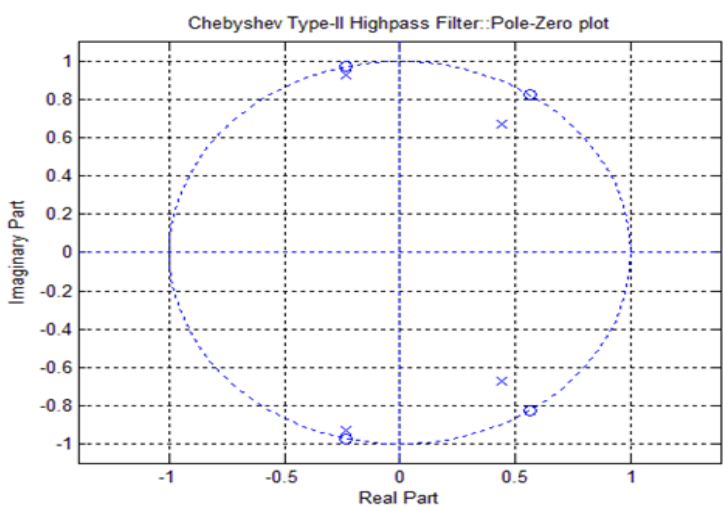

Fig.13Chebyshev(Type-II) Highpass filter pole-zero plot

\section{iv) Magnitude response (Filter order=6)}

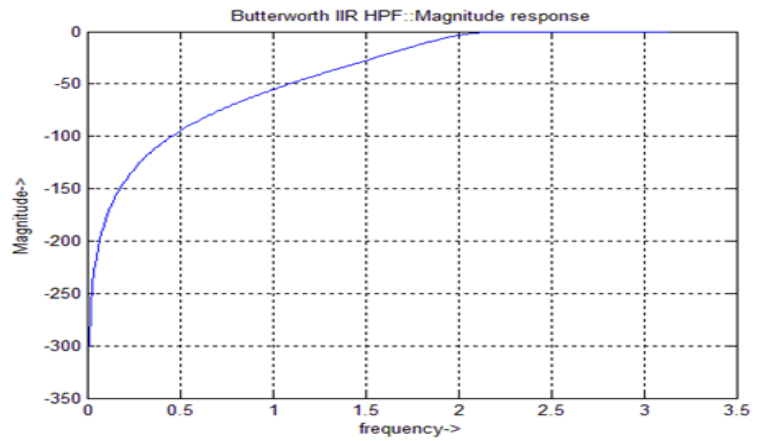

Fig.14 Butterworth Highpass filter Magnitude response

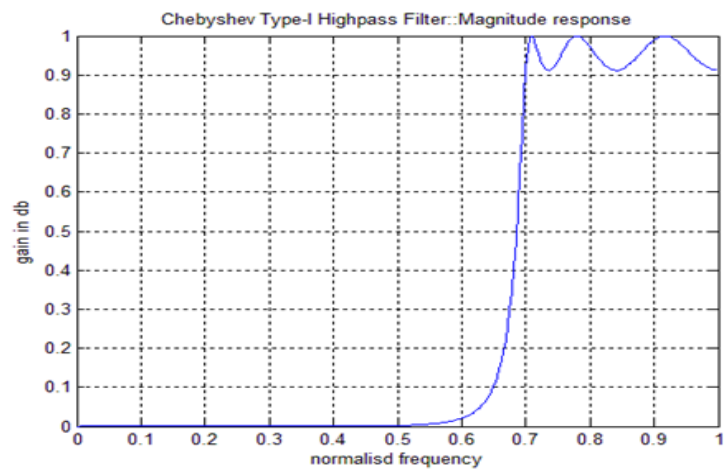

Fig.15 Chebyshev(Type-I) Highpass filter Magnitude response

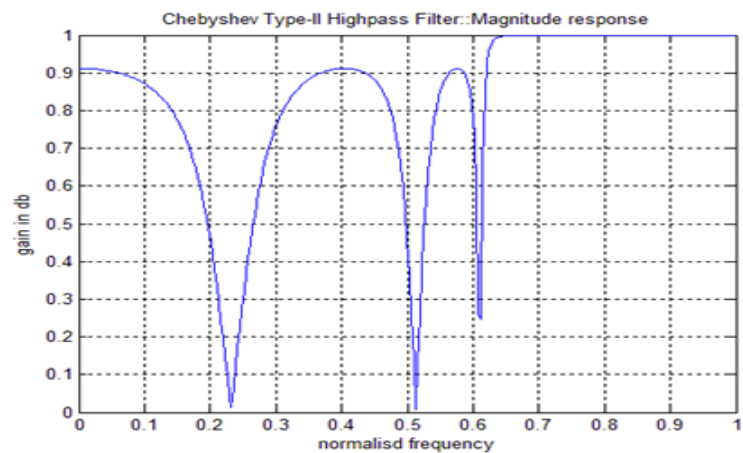

Fig.16Chebyshev(Type-II) Highpass filter Magnitude response

\section{v) Impulse response (Filter order =6)}

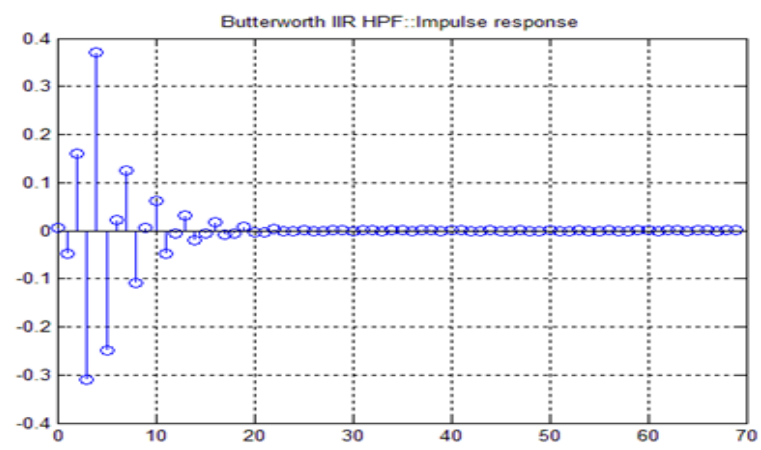

Fig.17 Butterworth Highpass filter Impulse response 


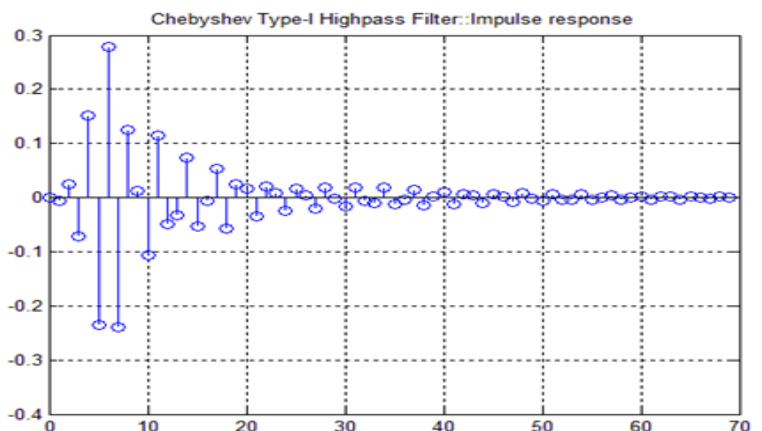

Fig.18Chebyshev(Type-I) Highpass filter Impulse response

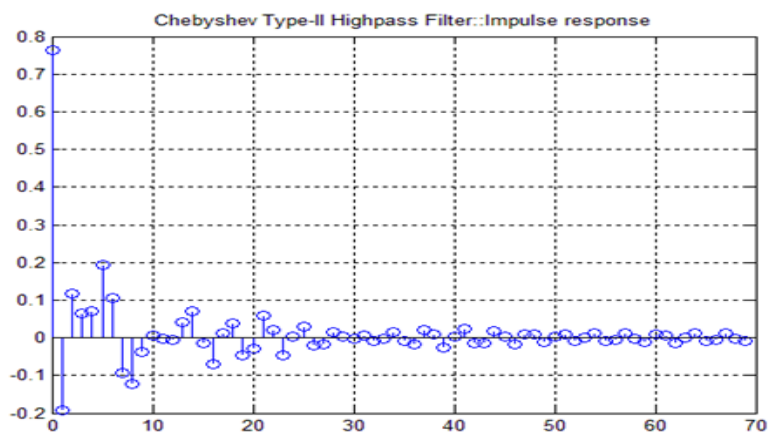

Fig.19Chebyshev(Type-II) Highpass filter Impulse response

\section{vi) Pole-Zero plot $($ Filter order $=6)$}

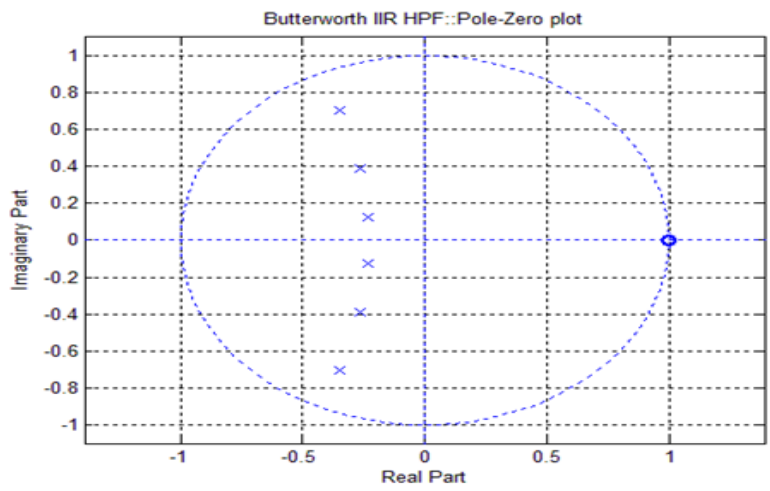

Fig.20 Butterworth Highpass filter pole-zero plot

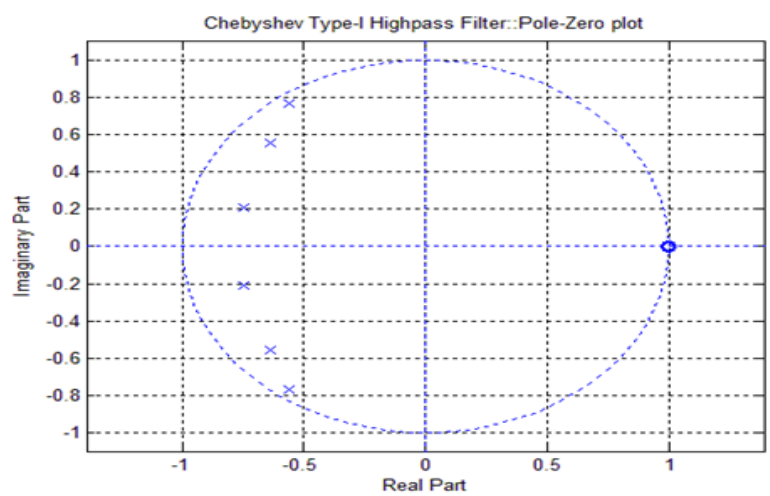

Fig.21 Chebyshev(Type-I) Highpass filter pole-zero plot

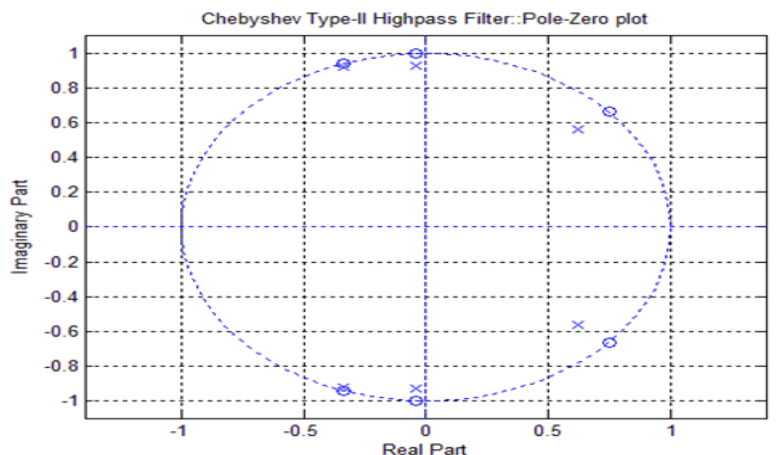

Fig.22 Chebyshev(Type-II) Highpass filter pole-zero plot

\section{Conclusion}

In this paper, the design and the calculation for coefficients of IIR Digital High pass filter are shown. The algorithm shown in Fig.4 helps us to determine the optimum coefficients of the IIR Digital High pass filter. Those coefficients are truly required to design the digital filter. After designing the IIR Digital High pass filter from the analog filter with the application of analog-to-digital mapping technique, we obtain the required coefficients as well as the magnitude response, impulse response and the pole-zero plot of the filter by simulating the necessary programs in Matlab7. The polezero plot shows the stability of the system. Here we can see that the IIR Digital High pass filter(Chebyshev Type-I, Chebyshev Type-II, Butterworth filter), designed with the application of the algorithm, are stable in nature. So, we can design a stable IIR Digital High pass filter by applying the algorithm.

\section{Reference}

[1] Kota Solomon Raju, Y.Pratap, Virendra Patel, S.M.M Naidu, Amit,Patwardhan and P.Bhanu Prasad, "Digital Multichannel GPS Receiver Baseband Modules using Model Based Design Tools" International Journal of Mobile Network Communications \& Telematics (IJMNCT) Vol.2, No.4, August 2012

[2] Behzad Razavi, Fellow, IEEE, "Cognitive Radio Design Challenges and Techniques", IEEE Journal of Solid-State circuits, vol. 45, no. 8, August 2010

[3] Asad A. Abidi, Fellow, IEEE, "The Path to the Software-Defined Radio Receiver", IEEE Journal of Solid-State circuits, vol. 42, no. 5, may 2007

[4] Subhadeep Chakraborty, Krishna Kumar Jha, Abhirup Patra, “ Design of IIR Digital Highpass Butterworth Filter using Analog to Digital Mapping Technique", International Journal of Computer Applications (0975 - 8887) Volume 52 - No. 7, August 2012

[5] Ranjit Singh and Sandeep K. Arya, "Determining Optimum coefficients of IIR Digital Filter using Analog to Digital Mapping," International Journal of Advancements in Computer Science and Information Technology, Vol. 01,No. 01, September 2011 pp.1923. 
[6]. R.S. Chauhan and Sandeep K. Arya,"Design of IIR digital filter using analog to digital mapping", Journal of Neural Computing Systems, Vol. 03,No. 01,2010,pp. 51-55.

[7] Ranjit Singh Chauhan and Sandeep Kumar Arya, " Determine Optimal Coefficients of IIR Digital Filters using Simulated Annealing", International Journal of Computer Applications (0975 - 8887) Volume 43- No.10, April 201236

[8]. P. Ramesh Babu,'Digital Signal Processing”, Fourth edition, Scitech Publication(India) Pvt. Ltd, Chennai,2008.

[9]. Ramakant A. Gayakwad, "Opamp and Linear Integrated Circuit", Fourth Edition, PHI Learning Private Limited, New Delhi, 2010.

[10] A. Sudhakar and Shyammohan S. Palli, "Circuits and Network", Fourth Edition, TataMcGraw Hill Education Private Limited, New Delhi, 2011.

[11] D. Chattopadhyay and P.C. Rakshit, "Fundamentals of Electric Circuit Theory", S.Chand \& Company Ltd. 2006.

[12] Johannes Innerbichler, $T U$ Graz, Achim Schweighofer, TU Graz, "Comparison of Characteristicsof FIR and IIR Filter", Journal of LATEX class filters, vol. 6, no. 1, January 2007.

[13] Gurleen Kaur and Ranjit Kaur, "Design of Recursive digital filters using Multiobjective Genetic algorithm", Gurleen Kaur et al. / International Journal of Engineering Science and Technology (IJEST), ISSN : 0975-5462 Vol. 3 No. 7 July 2011.

[14] Sangeetha Marikkannan, Leelavathi, Udhayasuriyan Kalaiselvi, Kavitha, "Scheduling and Allocation Algorithm for an Elliptic Filter", International Journal of Advanced Information Technology (IJAIT) Vol. 2, No.2, April 2012

[15] Nagendra Krishnapura, Abhishek Agrawal, and Sameer Singh, "A High-IIP3 Third-Order Elliptic Filter With Current-Efficient FeedforwardCompensated Opamps", IEEE Transactions on Circuit and System-II: Express Briefs, VOL. 58, NO. 4, APRIL 2011

[16] Proakis, J. G. and Manolakis, D. G. 2007. Digital Signal Processing: Principles, Algorithms, and Applications. Pearson Education Ltd.
[17] Sayan Ghosh, Debarati Kundu, Kaushik Suresh, Swagatam Das, and Ajith Abraham, "Design of Optimal Digital IIR Filters by Using a Bandwidth Adaptive Harmony Search Algorithm", 2009 World Congress on Nature \& Biologically Inspired Computing (NaBIC 2009)

[18] Nurhan Karaboga, "Digital IIR Filter Design Using Differential Evolution Algorithm", EURASIP Journal on Applied Signal Processing 2005:8, 1269-1276 (C)2005 Hindawi Publishing Corporation.

[19] Prof. SunayanaPatil, Pratik Pramod Bari, VivekAnandSakla, Rohit Ashok Shah, DharmilAshwin Shah, " Comparison of different realization techniques of IIR filters using system generator", International Journal of Engineering Research and Applications (IJERA) ISSN: 2248-9622.

[20].Samarjeet Singh and Uma Sharma, "Matlab based Digital IIR filter design", International Journal of Electronics and Computer Science Engineering, Vol. 01, No. 01,ISSN 2277-1956,pp.74-83.

[21].Yaduvir Singh, Sweta tripathi and Manoj Pandey, “ Analysis of Digital IIR filter with LabVIEW", International Journal of Computer Applications, Vol. 10, No. 06, 2010, pp.23-30.

\section{Authors Profile}

Subhadeep Chakraborty, born in 1986, is Assistant Professor in Calcutta Institute of Technology. He received the B.Tech degree from Saroj Mohan Institute of Technology, WBUT, India and M.Tech degree from Kalyani Govt. Engineering College, WBUT, India in Electronics and Communication Engineering in 2008 and 2010.The author has been teaching in Calcutta Institute of Technology for 2 years. His primary research interest includes Digital Signal Processing, Embedded System and Microprocessor.

Subhasish Maitra, is the Professor in Kalyani Govt. Engineering College in Electronics and Communication Engineering Department. The author has worked in the field of Signal processing and Cryptography for many years and his primary research interest includes Digital Signal Processing, Embedded System, Cryptography and Microprocessor . 